\title{
Towards the definition of a modelling framework for meaningful Human-loT Interactions
}

\author{
J. Waldo Cervantes-Solis, Chris Baber \\ Pervasive and Ubiquitous Computing Lab \\ School of Engineering \\ University of Birmingham \\ Birmingham, United Kingdom, B15 2TT
}

\begin{abstract}
A social-like environment for a meaningful Human-loT collaboration has been previously analysed from the perspective of conveying common goals amongst network participants. However, a clearly defined methodology for their implementation of physical systems is required. This work-inprogress paper provides an exploration of the TAFEI framework as a tool for the design and implementation of smart objects from a usability standpoint, in which a detailed description of a system and its user's interactions could be used by the system designer to architect better interfaces and interaction experiences, focusing on usability and opportunities for 'intelligent' loT behaviour.
\end{abstract}

HCl. Usability. Internet of Things. Task Analysis.

\section{INTRODUCTION}

In recent years Internet of Things (loT) devices have shifted from passive data collection objects, to things that actively engage with their entities, possessing enhanced capabilities that imbue them with varying degrees of agency and intelligence (Fortino, 2016).

Humans often collaborate with the loT both as beneficiary of their actions (Guo et al., 2011), and, in some form, as part of their control loops (Schirner et al., 2013). These interactions have resulted in a variety of approaches in which the IoT and its stakeholders organise and interact with each other.

With the objective to fill the gap in the design of 'smart objects' for the loT that promote meaningful Human-loT collaboration, this Work-in-Progress paper presents an exploration towards the development of a system modelling framework for the loT.

\section{BACKGROUND}

In Human-loT interaction, participants often create collaborative partnerships to achieve common goals in social-like structures (Nunes et al., 2015).

(Cervantes-Solis et al., 2015) analysed how 'smart objects' and humans collaborate towards common aims, emphasising goal achievement, over system's rules, highlighting the overarching purpose of the system. For human users, the anticipation of an loT system would be to obtain insight on the devices' operation, added convenience and comfort, richer user experiences or analytics. This expectation presumes a level of trust and common interests, much like social relationships, and as such, Human-loT interactions have been analysed from the perspective of sociallike organisational structures (Atzori et al., 2014), where two-way interactions occur, akin to a conversation amongst social actors (Cila et al., 2017).

This research focuses on providing an loT systemmodelling framework through which, by identifying and characterising the purpose of these conversations, meaningful Human-loT interactions could be attained.

Although modelling for the loT can be approached from different perspectives, from an usability point of view, (Matassa \& Simeoni, 2015) argues that when dealing with "objects whose natural functions" have been augmented by the loT, affordances "cannot immediately communicate to people their actual values and meanings". This can potentially create a detachment between the system's purpose and the user's actions and goals.

Thus, this work in progress paper aims to fill the gap in this disconnection, by analysing how tasks and goals are achieved in an instrumented object.

Hierarchical Task Analysis (HTA) (Stanton, 2006) and Task Analysis for Error Identification (TAFEI) (Baber \& Stanton, 1994, 1996) are tools developed to analyse and design for appropriate system usability through possible system actions available to users. Moreover, TAFEl's modelling methodology with the system's goal at its core, provides a suitable approach to analyse a system's usability, when it has been suggested that its precisely the system's goals that motivates the 
interaction, in the form of an overarching theme in this social-like collaboration between human and device (Cervantes-Solis \& Baber, 2016). Although TAFEI focuses on analysis for user error prediction, the technique provides a suitable approach to analyse novel forms of interactions on instrumented objects that could become different, but valid, goals not originally conceived in the system's design

\section{METHODS}

This study builds upon concepts developed in a previous experiment where an office's coffee making related appliances were instrumented in order to analyse how their interactions aggregated towards a common goal. It developed the notions of topics and themes as the conductive threads in the Human-loT collaborative environment (Cervantes-Solis \& Baber, 2016).

Modelling of meaningful interactions required analysing the system as a network with transitioning states, with each state describing the likelihood of the network being used for its original purpose, or if they describe an unexpected goal. As such, the TAFEl framework for modelling was used as a starting point for characterizing how the system's usability could lead to errors.

The experiment presented in (Cervantes-Solis \& Baber, 2016) focused on a test bed implemented with limited instrumentation, but supported by the objects affordances, providing information detailing solely to when the corresponding device became active.. The study's goal was to show how all devices participated towards a collective goal.

This paper focuses on the model for a single object in the network, exploring the potential deviations in operation that the instrumented appliance can take, and how they could impact the identification of topics and themes in the network. By providing higher granularity in an object's instrumentation, not only a higher detail of interactions could be identified as being part of a topic or theme, but would also provide a means of describing additional topics and themes not previously considered in the original system's design.

\subsection{A worked example}

A coffee machine was instrumented with sensors to detect button presses, displacement of the empty coffee capsules canister and water tank, its temperature, and operation of the handle to place coffee capsules for coffee preparation.

Each instrumented part of the object presents a point of user interaction, and for TAFEI, represents the system image for analysis.

The TAFEI methodology requires the definition of specific user goals, with the intention of constraining the number of possible states. As mentioned, the notion of an association between user and loT system is the focus of this study, as such, TAFEl's goals are interpreted in this paper as the theme of the collaboration. Hence, the theme defined for this coffee machine is:

\section{- THEME_1: Make a cup of coffee}

Following decomposition of the themes in terms of the states the object's passes to complete it, and the definition of plans through HTA, Table 1 shows a transition matrix for the analysed system, derived from the state and HTA diagrams. Legal transitions (marked as L) for the 'coffee making' theme are shown in the main diagonal. TAFEI is generally used to find possible errors in product usability design. However, in the case of an instrumented device with the potential of being imbued with a degree of intelligence, such as the one presented in this paper, it becomes useful to identify other interactions that could enable different goals or themes that could provide useful information to the user. The transition matrix shows the valid path towards the specified theme, whilst also providing information on the illegal (I) transitions in the context of said goal, as well as those that are impossible (shown in table 1 as "-') As such, another theme could be defined:

\section{- THEME_2: Coffee machine servicing.}

Table 1. Transition matrix for 'coffee making' theme. Highlighted in green is the path leading for the successful completion of the goal. In yellow, although invalid for the analysed theme, an emergent secondary theme is enabled, showing the required path for 'servicing' the coffee machine.

\begin{tabular}{|c|c|c|c|c|c|c|c|c|}
\hline & \multicolumn{8}{|c|}{ To State } \\
\hline \multirow{8}{*}{$\begin{array}{l}\text { From } \\
\text { State }\end{array}$} & & 0 & 1 & 2 & 3 & 4 & 5 & 6 \\
\hline & 0 & I & $\mathrm{L}$ & - & - & - & - & I \\
\hline & 1 & - & - & L & I & - & - & I \\
\hline & 2 & I & - & I & $\mathrm{L}$ & I & - & I \\
\hline & 3 & I & - & I & I & L & - & I \\
\hline & 4 & - & - & - & - & I & $\mathrm{L}$ & - \\
\hline & 5 & I & - & - & I & $\mathrm{L}$ & I & $\mathrm{L}$ \\
\hline & 6 & I & I & I & I & - & - & I \\
\hline
\end{tabular}

\section{DISCUSSION}

TAFEI was originally conceived as a goal based modelling framework, allowing identification of invalid actions or goals that possibly were 
necessarily not part of the original design. Hence, it supports a product's design and implementation process to consider circumstances in which unexpected object or user behaviour could hinder or modify its usability.

For the IOT, TAFEI is repurposed to model ways in which the system's functionality could be extended. In contrast to the system used in the experiment that motivated this research, in which objects were only instrumented to detect whether they were used or not, higher granularity in sensor instrumentation allowed a thorough exploration of a device's states and transitions. Hence, it would be possible identify other topics (sub-goals or actions) and thus, present additional knowledge to the user. For example, in the described scenario, the coffee making theme for the coffee machine (it's main goal) is clear, with precisely defined states, plans and transitions.

From a device perspective, in a scenario of a 'dumb' coffee machine, the definition of all possible themes enabled by the system might not be relevant, as it will only carry on with its expected functionality without actually extending its capabilities. However, for a 'smart' machine, the extension of a 'servicing' theme could be used to allow the machine to pre-emptively notify the user whether the machine needs descaling (by keeping track of the number of times the water has been replaced and the amount of coffees made), or that the empty capsules canister needs emptying. This implication would in fact enable the degree of intelligence pursued by most loT ecosystems.

From an usability standpoint, a more detailed description of a system and its user's interactions could be used by the system designer to architect better interfaces and interaction experiences, focusing on usability and opportunities for 'intelligent' IoT behaviour.

As a work-in-progress, further development needs to be accomplished; mainly to address the issue of how the system would be able to identify which goal is which when more than one is present. Fully automated decision making nodes need to be implemented to evaluate when and which notifications could be made to the user, taking into consideration results and data from previous machine learning efforts.

Supplementary activities need to be carried out towards establishing a clearly defined framework, and to test and measure its capacity to properly describe and define a meaningful Human-loT collaboration in a social-like environment, capable of managing its themes.

\section{ACKNOWLEDGEMENTS}

The work reported in this paper is partly supported by Consejo Nacional de Ciencia y Tecnología (National Council of Science and Technology), CONACyT, in Mexico. We also appreciate the support from Universidad Iberoamericana, Mexico City, Mexico.

\section{REFERENCES}

Atzori, L., Carboni, D., \& lera, A. (2014). Smart things in the social loop: Paradigms, technologies, and potentials. Ad Hoc Networks, 18, 121-132. Elsevier B.V.

Baber, C., \& Stanton, N. A. (1994). Task analysis for error identification: a methodology for designing error-tolerant consumer products. Ergonomics, 37(11), 1923-1941.

Baber, C., \& Stanton, N. A. (1996). Human error identification techniques applied to public technology: Predictions compared with observed use. Applied Ergonomics, 27(2), 119-131.

Cervantes-Solis, J. W., \& Baber, C. (2016). Towards Theme Discovery Paradigm in the Internet of Things. In P. Waterson, R. Sims, \& E.-M. Hubbard (Eds.), Contemporary Ergonomics and Human Factors 2016 (pp. 335-340). Chartered Institute of Ergonomics \& Human Factors.

Cervantes-Solis, J. W., Baber, C., Khattab, A., \& Mitch, R. (2015). Rule and Theme Discovery in Human Interactions with an "Internet of Things." Proceedings of the British HCl 2015 Conference.

Cila, N., Smit, I., Giaccardi, E., \& Kröse, B. (2017). Products as Agents. Proceedings of the 2017 CHI Conference on Human Factors in Computing Systems - CHI '17 (pp. 448-459). New York, New York, USA: ACM Press.

Fortino, G. (2016). Agents Meet the loT: Toward Ecosystems of Networked Smart Objects. IEEE Systems, Man, and Cybernetics Magazine, 2(2), 43-47.

Guo, B., Zhang, D., \& Wang, Z. (2011). Living with Internet of Things: The Emergence of Embedded Intelligence. 2011 International Conference on Internet of Things and 4th International Conference on Cyber, Physical and Social Computing, 297-304. leee.

Matassa, A., \& Simeoni, R. (2015). Eliciting affordances for smart objects in loT Era. Lecture Notes of the Institute for Computer Sciences, Social-Informatics and Telecommunications Engineering, LNICST, 150(October 2014), 77-81.

Nunes, D., Zhang, P., \& Silva, J. (2015). A survey 
on Human-in-the-Loop applications towards an Internet of All. IEEE Communications

Surveys \& Tutorials, 17(X), 1-1.

Schirner, G., Erdgogmus, D., Chowdhury, K., \&

Padir, T. (2013). The Future of Human- in-theLoop Cyber-Physical Systems.

ieeexplore.ieee.org.

Stanton, N. A. (2006). Hierarchical task analysis:

Developments, applications, and extensions.

Applied ergonomics, 37(1), 55-79. Elsevier. 